\title{
Critical illness polyneuropathy
}

\author{
Mahadevan Sugunadevan $^{1^{*}}$, PrageethTillekeratne ${ }^{2}$ \\ Consultant Anaesthetist ${ }^{1}$, Resident House Officer, Intensive care ${ }^{2}$, Base Hospital, Puttalam, Sri \\ Lanka.
}

*Corresponding author: mahasugunan@gmail.com

\begin{abstract}
Acquired neuromuscular disorder due to critical illness polyneuropathy (CIP) develops in patients admitted to Intensive care unit for more than one week. CIP could present with weakness and delayed weaning from mechanical ventilation. It is often diagnosed on clinical suspicion and confirmed with electro-physiological studies. Severe sepsis and multi-organ failure are important risk factors and impaired glycaemic control contributes to $\mathrm{CIP}^{1}$. CIP results in prolonged mechanical ventilation, prolonged rehabilitation and increased morbidity and mortality.
\end{abstract}

Keywords:

Critical illness polyneuropathy (CIP): weakness: delayed weaning: rehabilitation

\section{Case Report}

61 year old lady, a known patient with type II diabetes mellitus, bronchial asthma and essential hypertension was admitted to a medical ward with productive cough, pleuritic type of chest pain and difficulty in breathing. She was treated as for lower respiratory tract infection with antibiotics, and regular nebulisation with salbutamol and ipratropium solutions.

As she was desaturating despite oxygen via face mask she was transferred to intensive care unit for respiratory support. On arrival at the intensive care unit she was dyspnoeic, cyanosed, tachycardic, tachypnoeic and had bilateral crepitations. Chest x-ray showed bilateral patchy consolidation. She was electively intubated and ventilated on pressure control mode. Internal jugular cannulation was performed on the right side. She had the features of severe sepsis. She was adequately fluid resuscitated and started on noradrenalin and dobutamine. She was treated with antibiotics after obtaining cultures. Her glycaemic control was poor and she was treated with insulin infusion and later switched to regular dose of long acting insulin. Patient didn't receive any steroids or neuromuscular blocking agents during the intensive care unit stay.

Her clinical symptoms improved over 4-5 days and she was weaned off inotrope support. It was noticed that she had marked weakness in the lower limbs and upper limbs. She had reduced power in her limbs with loss of tendon reflexes. Despite clinical improvement, her effort for spontaneous respiration was poor. Therefore, weaning from mechanical ventilation was difficult. As she was difficult to wean from ventilation, she had an elective tracheostomy done.

The CPK/CPKMB was normal and she was referred for neurological assessment. She had a CT scan of the head and EEG which were normal. Lumbar puncture studies were normal. She had electrophysiological studies which confirmed critical illness polyneuropathy.

She was given physiotherapy regularly. Glycaemic control was with insulin therapy. Her septic focus was aggressively treated. She had naso-gastric feeding with high protein diet for nutritional support. She improved gradually. We could manage to wean her from ventilation after 21 days of mechanical ventilatory support. She was mobilised and eventually discharged to the ward.

\section{Discussion:}

Acquired neuromuscular weakness due to critical illness neuropathy is not uncommon in patients who are admitted to intensive care unit with critical illness for more than one week. Prospective studies have shown about 25-60\% of patients on mechanical ventilator for more than one week develops critical illness neuropathy ${ }^{2}$. It is twice commoner in males and age more than 50 years. It can occur in all age groups but is rare in the paediatric age group ${ }^{2}$. It often presents with muscle weakness and difficulty in weaning from mechanical 
ventilation. It is often diagnosed on clinical suspicion and confirmed with electrophysiological studies.

Risk factors for critical illness neuropathy are severe sepsis, presence of multi-organ dysfunction, poor glycaemic control, use of high dose of steroids, excess usage of amino glycosides and neuromuscular blocking agents ${ }^{3}$. Presence of hypoxia, hypotension and hyperpyrexia have contributed as risk factors to critical illness neuropathy. The following factors have been found as independent risk factors like use of total parental nutrition, duration of organ dysfunction, severity of illness, renal impairment, need for renal replacement therapy, hyper osmolality, low serum albumin and need for vasopressor support ${ }^{4}$.

Critical illness neuropathy was first described by Bolton and colleagues in $1986^{3}$. Patients with critical illness neuropathy presents with flaccid symmetrical weakness predominantly of the lower limbs. Upper limb involvement is not uncommon. Reduction or absence of tendon reflexes is often seen ${ }^{1}$. Critical illness neuropathy often presents with weakness of the peripheral nerves. But it could have central nervous involvement with diffuse encephalopathy ${ }^{5}$.

Pathophysiology of critical illness neuropathy is complex and unclear. Bolton and colleagues have hypothesised that sepsis related disturbances in the micro-circulation of peripheral nerves and muscles is a crucial event in the pathogenesis of CIP. This may be mediated by enhanced expression of E-selectin in vascular endothelium of the peripheral nerves ${ }^{6}$. Cytokines produced can cause increased micro-vascular permeability and causes neuronal oedema. This leads to neuronal hypoxia and axonal degeneration ${ }^{6}$. Hyperglycaemia and cytokines can cause impairment of the microcirculation of the nerves causing neuropathy. It is associated with primary axonal degeneration. There is impaired scavenging of reactive oxygen species $^{6}$ contributing to bio-energetic failure. Enhanced permeability to cytokines leads to direct tissue injury. Protein catabolism and muscle wasting is noticed due to proteolysis pathway involvement with calpain ${ }^{6}$. Calpain is calcium activated proteases, which alters cellular calcium homeostasis due to endotoxaemia and inflammation ${ }^{6}$. There is relative deficiency of glutamate which enhances protein synthesis and prevents proteolysis. Increased catabolic hormones and decreased anabolic hormones contribute to myofilament loss and apoptosis ${ }^{6}$. Interaction of lipopolysaccharides with voltage gated sodium channels causes muscle membrane in-excitability. ${ }^{6}$

Nerve conduction and electro myographic (EMG) studies reveal reduction in amplitude and duration of muscle action potentials. Reduced amplitude of sensory and motor action potentials is also noticed due to axonal oedema ${ }^{2}$. They show normal motor velocities, response to repetitive stimulations and distal motor latencies. Laboratory blood tests are not diagnostic. They show normal or marginally elevated creatine phospokinase levels ${ }^{2}$.

Differential diagnosis for critical illness polyneuropathies includes acute spinal cord lesions, Gullien Barre syndrome, myasthenia gravis, and electrolyte disturbances like hypokalaemia, hyponatraemia and chronic inflammatory demyelinating poly-neuropathy.

Critical illness polyneuropathy has many impacts to the patients. They cause muscle weakness and paralysis and delayed rehabilitation. They cause prolonged weaning from mechanical ventilation. It causes prolonged ICU and hospital stay. It is associated with complications related to prolonged immobilisation like pneumonia, deep vein thrombosis, and pulmonary embolism. The recovery is characterised by re-innervations to muscles and restoration of sensory function ${ }^{4}$. Full recovery is reported in $50 \%$ of the patients ${ }^{4}$. However, rest of the patients shows partial recovery and no recovery at all. The presence of tetraplegia, severe ARDS, poor glycaemic control and severe sepsis increases poor outcome ${ }^{2}$.

Prevention of CIP is very important. Preventing any additional pressure neuropathies should be avoided. Careful positioning to prevent pressure sores is very vital. Aggressive treatment of sepsis and tight glycaemic control is very important. Several specific therapies have been mentioned theoretically like anti-oxidant therapy, use of immunoglobulins, growth hormones and testosterones supplements ${ }^{7}$. But none of these have shown beneficial effects ${ }^{7}$. Proper nutritional schemes with nutritional 
interventions and supplementations are very important in the management of CIP. Tight glycaemic control with insulin therapy has shown a good evidence of improvement. Insulin has the potential beneficial effects like antiinflammatory effect $^{8}$, endothelial protection ${ }^{3}$, and improvement of dyslipidaemia and neuroprotective effects. Many studies of tight glycaemic control with insulin have shown better outcome of CIP.

In summary, CIP is not an uncommon illness in critically ill patients. Early control of severe sepsis and having tight control of blood glucose levels are important to prevent the severity of the disease. Considering the physical and social impact after the development of the disease it is important to take adequate precautions to prevent the development of CIP.

\section{References}

1. Bednarick J, Lukas Z, Vondracek P. Critical illness polyneuromyopathy: the electrophysiological components of a complex entity. Intensive Care Med 2003; 29:1505-1514. http://dx.doi.org/10.1007/s00134-003-1858-0 PMid: 12879242

2. Bolton CF. Sepsis and the systemic inflammatory response syndrome: neuromuscular manifestations. Critical Care Medicine. 1996; 24: 1408-1416. http://dx.doi.org/10.1097/00003246-199608000$\underline{00022}$

PMid:8706499

3. Visser LH. Critical illness polyneuropathy and myopathy: clinical features, risk factors and prognosis. European Journal of Neurology 2006: 13: 1203-1212.

http://dx.doi.org/10.1111/j.14681331.2006.01498.x PMid: 17038033

4. Khilnani G, Pandit J N. Critical illness polyneuropathy. Journal of Associate Physicians India. 1997: 45: 732-733

5. Van den Berghe G, Wouters P, et al. Intensive Insulin Therapy in Critically Ill Patients. N Engl J Med 2001; 345:1359-1367 http://dx.doi.org/10.1056/NEJMoa011300 PMid:11794168

6. Hermans G, De Jonghe B, Bruyninckx F, Van den Berghe G. Clinical review: Critical illness polyneuropathy and myopathy. Critical care 2008: $\quad$ 12: 238-248 http://dx.doi.org/10.1186/cc7100 PMid:19040777 PMCid:PMC2646339

7. Herridge MS, Cheung AM et al. One-Year Outcomes in Survivors of the Acute Respiratory Distress Syndrome. New England Journal of
Medicine 2003:348: 683-693 http://dx.doi.org/10.1056/NEJMoa022450 PMid: 12594312

8. Hund E, Genzwurker $\mathrm{H}$ et al. Predominant involvement of motor fibres in patients with critical illness polyneuropathy. British Journal of Anaesthesia $\quad 1997 ; \quad 78: \quad 274-278$ http://dx.doi.org/10.1093/bja/78.3.274 